\title{
Microscopic Colitis A Commonly Missed Diagnosis that Needs Further Studies and Thorough Investigations
}

\section{Omar S Mansour*}

Consultant Colorectal and Laparoscopic General Surgeon, Assistant Professor and Clinical Lecturer in General Surgery, Department of Surgery, School of Medicine, Al Balqa Applied University, Al Salt, Jordan

*Corresponding Author: Omar S Mansour, Consultant Colorectal and Laparoscopic General Surgeon, Assistant Professor and Clinical Lecturer in General Surgery, Department of Surgery, School of Medicine, Al Balqa Applied University, Al Salt, Jordan.

DOI: 10.31080/ASGIS.2020.03.0117
Received: January 27, 2020

Published: February 01, 2020

C All rights are reserved by $\mathbf{0 m a r} \mathbf{S}$

\section{Mansour.}

\begin{abstract}
Microscopic colitis is a chronic inflammatory disease of the colon which is characterized by chronic diarrhea, normal colonic mucosa on colonoscopy and abnormal histological findings on random colonic biopsies. When referred for lower GI endoscopy, these patients have normal colonic mucosa on their first colonoscopy but not all of them get random colonic biopsies done. Provided that microscopic colitis has a prevalence of around 48 to 219 per 100,000 and is diagnosed in around 7.5\% of all patients undergoing evaluation for chronic diarrhea, it would be strongly advised that all patients with chronic or recurring diarrhea and abdominal pain to get random colonic biopsies during this first lower GI endoscopy even if they are found to have normal colonic mucosa. This approach would have too goals. The first goal is to get the proper diagnosis on their first visit. The second goal is to prevent further colonoscopies and their potential costs and complications if these patient's symptoms do recur in the future without any proper diagnoses after being flagged with functional abdominal pain and normal colonoscopy. Finally, current guidelines from the American Gastroenterological Association Institute on the medical management of microscopic colitis which were published in 2016 are clear and supported by strong evidence [11].
\end{abstract}

Keywords: Microscopic Colitis, Abdominal Pain, Diarrhoea, Colonoscopy Random Biopsy, Guidelines

There has been a rise in the number of patients seeking medical evaluation for recurrent and chronic abdominal pain, bloating and change in bowel habits. Most of these patients are usually diagnosed by their primary physicians with functional abdominal pain (FAP) which includes irritable bowel syndrome (IBS). When referred for lower GI endoscopy, some of these patients have normal colonic mucosa on their first colonoscopy. Not all of these patients get random colonic biopsies done. For those who get their colons biopsied, many of them are diagnosed with different types of colitides like microscopic colitis and inflammatory bowel disease. Microscopic colitis is diagnosed in around $7.5 \%$ of all patients undergoing evaluation for chronic diarrhea. The reported prevalence of microscopic colitis ranges from 48 to 219 per 100,000 inhabitants [1].

Microscopic colitis (MC) is a chronic inflammatory condition characterized by chronic diarrhea, normal colonic mucosa, and abnormal histologic findings. There have been two types of microscopic colitis described in the literature, collagenous colitis (CC) and lymphocytic colitis (LC). Features of microscopic collagenous colitis (CC) were first described by Lindström in 1976 [2]. In 1989, Lazenby and colleagues described features of lymphocytic colitis (LC) [3].
A recent French study reported that the incidence of MC was 7.9 per 100,000 inhabitants, similar to the incidence of Crohn's disease in that population [4].

Risk factors for microscopic colitis include female gender. The ratio of women to men is 2.4 to 1 and women have a proportionally greater risk of collagenous colitis [5]. Current smoking increases the risk of persistent disease [6]. A history of autoimmune disease, including diabetes mellitus, rheumatoid arthritis, and celiac sprue, is associated with MC [7].

Celiac disease is associated with a 50 to 70 times greater risk of MC, especially in middle-aged women, and with severe villous atrophy [8]. Established risk factors also include increasing age, with $75 \%$ of the people affected being over 50 years old [9].

Medications associated with microscopic colitis include nonsteroidal anti-inflammatory drugs (NSAIDs) such as aspirin; selective serotonin reuptake inhibitors; proton pump inhibitors (PPIs); and drugs with various other mechanisms of action, including statins, beta blockers, angiotensin-converting enzyme inhibitors, and angiotensin receptor blockers [10]. 
Microscopic colitis can have a chronic, intermittent or chronicrecurrent course. Associated symptoms are weight loss (seen in $42 \%$ of patients), abdominal pain (41\%) and nocturnal diarrhoea (27\%), and quality of life (QoL) may be significantly impaired [9]

Symptoms may vary from mild chronic watery diarrhea to incapacitating, increased stool frequency with urgency and fecal incontinence [7] Approximately half of the affected population will report some weight loss or abdominal pain [5] Approximately $50 \%$ of MC patients have mild anemia and mildly elevated inflammatory markers [9].

There has been no evidence to suggest that microscopic colitis is associated with increased mortality. Despite that, symptoms can lead to impaired quality of life. Unlike other types of inflammatory colitides, there is no evidence in the literature to prove that the chronicity of colonic inflammatory changes in microscopic colitis increases long-term unfavorable outcomes such as colorectal cancer, inflammatory bowel disease or any need for colonic surgery [11].

A new entity of microscopic colitis called tingible body colitis was described recently in an original article. According to this study, all patients involved had normal colonoscopy findings. They all had random colonic biopsies taken during their colonoscopies. Each colonic specimen was examined histopathologic ally for the presence of tingible-body macrophages in the subepithelial surface layer, features of glandular distortion, and features of activity [12].

Chronic abdominal pain is a leading cause for referrals to the GI clinics. Many of these referrals are diagnosed as Functional Bowel Disorders. Functional bowel disorders, as a diagnostic entity, account for in excess of one-third of new patient referrals to gastroenterology clinics in secondary care of the National Health Service (NHS) and represent a heterogeneous group of disorders. Although seemingly similar to IBS, the functional abdominal pain (FAP) syndrome is characterized by frequent or continuous abdominal pain associated with a degree of loss of daily activity, in the absence in change in bowel habit. This has been illustrated in the Third Rome Criteria [13]

After proper work up and when the random biopsies of the colon come back to be normal, these patients are usually diagnosed with Functional abdominal pain (FAP). FAP has a UK reported population prevalence of between $0.5 \%$ and $1.7 \%$, with a female preponderance [14].

Microscopic colitis is defined as chronic inflammatory bowel disease characterized by chronic watery diarrhea and diagnosed with the histologic hallmarks of disease despite a macroscopically normal large bowel [15].
According to the American Gastroenterological Association (AGA) institute guideline on the medical management of microscopic colitis the following recommendations were drawn [11]. In patients with symptomatic microscopic colitis, the AGA recommends treatment with budesonide over no treatment for the induction of clinical remission (moderate quality of evidence). Also in patients with symptomatic microscopic colitis, the AGA recommends treatment with budesonide over mesalamine for the induction of clinical remission (strong recommendation, high quality of evidence). In patients with symptomatic microscopic colitis in whom budesonide therapy is not feasible, the AGA suggests treatment with mesalamine over no treatment for the induction of clinical remission (conditional recommendation, moderate quality of evidence). A high-quality clinical trial provided direct evidence that budesonide should be considered first-line therapy over mesalamine whenever possible. Patients with symptomatic microscopic colitis who were treated with budesonide $9 \mathrm{mg}$ daily were almost twice as likely as those treated with mesalamine $3 \mathrm{~g}$ daily to successfully achieve clinical and histological remission, and there was no statistically significant difference in occurrence of any adverse events [11].

Finally, for patients with recurrence of symptoms following discontinuation of induction therapy for microscopic colitis, the AGA recommends budesonide for maintenance of clinical remission (strong recommendation, moderate quality of evidence).

\section{Conclusion}

MC is present in around $10 \%$ of people with diarrhoea-predominant irritable bowel syndrome and 7.5 - 10\% of those with chronic diarrhea. The newly described entity of tangible body colitis, though not yet validated, would draw our attention to the unrecognized and uncategorized types of recurrent colonic mucosal inflammatory changes that would need to be further studied and deeply investigated. This would make our clinical outcome and diagnoses easier and would lead to better patients understanding of the underlying pathology and facilitate successful treatments.

Guidelines from the American Gastroenterological Association Institute on the medical management of microscopic colitis which were published in 2016, are clear and supported by strong evidence. We should follow those guidelines in our management of proven microscopic colitis cases.

Finally, and at our university, we are currently undertaking a retrospective analysis of patients with chronic and recurring abdominal pain and erratic bowel habits who had colonoscopies and random colonic biopsies done. The aim of this study is to look for risk factors for microscopic colitis, investigate patients' satisfaction with their treatments, and to validate the diagnoses of microscopic colitis, including the newly described tangible body colitis, and the efficacy of treatments given to those patients. 


\section{Bibliography}

1. Pardi DS., et al. "American Gastroenterological Association institute technical review on the medical management of microscopic colitis". Gastroenterology 150 (2016): 247-274.

2. Lindström CG. 'Collagenous colitis' with watery diarrhoea-a new entity?" European Journal of Plant Pathology 11.1 (1976): 87-89.

3. Lazenby AJ., et al. "Lymphocytic ("microscopic") colitis: a comparative histopathologic study with particular reference to collagenous colitis". Human Pathology 20.1 (1989): 18-28.

4. Fumery M., et al. "Incidence, Clinical Presentation, and Associated Factors of Microscopic Colitis in Northern France: A Population-Based Study". Digestive Diseases and Sciences 62.6 (2017):1571-1579.

5. Olesen M., et al. "Lymphocytic colitis: a retrospective clinical study of 199 Swedish patients”. Gut 53.4 (2004): 536-541.

6. Roth B., et al. "Smoking- and alcohol habits in relation to the clinical picture of women with microscopic colitis compared to controls". BMC Womens Health 14 (2014): 16.

7. Macaigne G., et al. "Microscopic colitis or functional bowel disease with diarrhea: a French prospective multicenter study". The American Journal of Gastroenterology 109.9 (2014): 14611470.

8. Stewart M., et al. "The association of coeliac disease and microscopic colitis: a large population-based study". Alimentary Pharmacology and Therapeutics 33.12 (2011): 1340-1349.

9. Bohr J., et al. "Collagenous colitis: a retrospective study of clinical presentation and treatment in 163 patients". Gut 39.6 (1996): 846-851.

10. Masclee GM., et al. "Increased risk of microscopic colitis with use of proton pump inhibitors and non-steroidal anti-inflammatory drugs". The American Journal of Gastroenterology 110.5 (2015): 749-759.

11. Colitis., et al. "American Gastroenterological Association Institute Guideline on the Medical Management of Microscopic". Gastroenterology 150.1 (2016): 242-246.

12. Sohaih Abu-Farsakh., et al. "Tingible Body Colitis: A Novel Entity of Colitis Causing Diarrhea". American Journal of Clinical Pathology 138.1 (2012): A098.

13. Drossman DA. "Rome III: the functional gastrointestinal disorders, 3rd ed". McLean, VA: Degnon Associates (2006):1048.

14. Adam D Farmer and Qasim Aziz. "Mechanisms and management of functional abdominal pain". Journal of the Royal Society of Medicine 107.9 (2014): 347-354.
15. Karen Boland and Geoffrey C Nguyen. "Microscopic Colitis: A Review of Collagenous and Lymphocytic Colitis". Gastroenterology and Hepatology 13.11 (2017): 671-677.

\section{Assets from publication with us}

- Prompt Acknowledgement after receiving the article

- Thorough Double blinded peer review

- Rapid Publication

- Issue of Publication Certificate

- High visibility of your Published work

Website: www.actascientific.com

Submit Article: www.actascientific.com/submission.php Email us: editor@actascientific.com

Contact us: +919182824667 